\title{
A DIMENSÃO DO INVENTÁRIO DE PAPÉIS SEXUAIS (BSRI): A MASCULINIDADE E FEMINILIDADE EM UNIVERSITÁRIOS*
}

\author{
THE DIMENSION IN THE INVENTORY OF SEXUAL \\ ROLES OF BEM (BSRI): THE MASCULINITY \\ AND FEMININITY ON UNIVERSITARIES
}

\author{
Nilton Soares FORMIGA \\ Leoncio CAMINO \\ Universidade Federal da Paraíba
}

\begin{abstract}
RESUMO
Este trabalho aborda as concepções da feminilidade e masculinidade da escala BSRI de Bem (1974). Discute na identidade de gênero as existências de características especificam que salientam o expressar e comportar-se de ambos - homem e mulher. Assim, a dimensão de feminilidade refere-se mais a estereótipo que a disposição individual, possuindo traços, como: o apoio afetivo as outras pessoas, etc. apresentando expressividade; a dimensão masculinidade apresenta as características: necessidade de realização, agressividade, dominância e etc. uma maior instrumentalidade. Assim, pretende-se estudar a maneira em que estas duas dimensões se encontram para os universitários. A pesquisa foi realizada em 423 sujeitos, entre as universidades públicas e privadas de João Pessoa, nos cursos de ciências humanas. Os sujeitos responderam a escala BSRI de Bem, entre outras variáveis. Observou-se que as mulheres apresentam maior freqüência na dimensão de feminilidade e os homens na de masculinidade. Conclui-se que, os sujeitos percebem tais dimensões de forma estereotipada, generalizando as características para ambos os gêneros.
\end{abstract}

Palavras-Chave: Masculinidade; Feminilidade; Sexualidade.

\section{ABSTRACT}

This work approaches the conceptions of the femininity and manliness of the scale BSRI of Bem (1974). It Discusses in the gender identity the

\footnotetext{
(*) Nota dos autores: Durante a realização deste estudo, o autor contou com Bolsa de IC/CNPq, instituição a qual agradece. Endereço para correspondência: Av. Sapé, 1267, Apto 1502, CEP 58038382, João Pessoa, PB. E-mail: nsformiga@yahoo.com.
} 


\begin{abstract}
existences of characteristics that points out the to express and to behave of both - man and woman. Thus, the femininity dimension refers more to stereotype that the individual disposition, possessing lines, as: the affective support the other people, etc. presenting expressiveness; the dimension masculinity presents the characteristics: accomplishment need, aggressiveness, dominance and etc. a larger instrumentality. Thus, intends to study the way in that these two dimensions meet for the university students. The research was accomplished in 423 subjects, among the public and private universities of João Pessoa, in the courses scientific humans. The subjects answered the scale BSRI of Bem, between another variables. It was observed that the women present larger frequency in the femininity dimension and the men in the one of masculinity. Conclude to be, that the subjects notice such dimensions in a stereotyped way, generalizing the characteristics for both goods.
\end{abstract}

Key Words: Masculity; Femininity; Sexuality.

\section{INTRODUÇÃO}

Muito tem sido discutido a respeito da diferenciação de gênero, seja em termos fisiológicos, psicológicos ou cognitivos. É de comum acordo que a identidade de gênero não se deve a um único fator, assim, os mecanismos cognoscitivos e os processos informativos do contexto social são capazes de desenvolver um esquema de gênero refletindo as crenças existentes numa determinada sociedade, relacionando tanto as características e papéis manifestados por homens e mulheres quanto uma auto-imagem que direciona os comportamentos, atitudes e as características esperada pela sociedade. (Baron, 1996; Bem, 1981).

As pesquisas sobre masculinidade $\mathrm{e}$ feminilidade têm apresentado dimensões independentes, que inclui aparência, comportamento, personalidade e interesse, desta forma, pode-se argumentar desde aos aspectos de gênero aos traços de personalidade, atribuindo uma instrumentalidade - assertivo, independente, etc. - ao homem e expressividade - simpática, carinhosa, etc. - a mulher (ver Manstead \& Hewstone, 1995; Ortiz \& Valencia, 1998). Desta maneira, tal relação - instrumentalidade vs expressividade - e a divisão dos papéis entre os sexos, atribuem ao homem qualidades orientadas ao êxito, a independência, a afirmação, a decisão e necessidade de realização, permitindo que o seu desempenho tanto no ambiente familiar quanto extrafamiliar, ocupe o papel de líder, como também, características do tipo agressivo, dominante, aventureiro; quanto as mulheres se atribui qualidades que as orientem ao contato interpessoal, a amabilidade com os outros, necessidade de afiliação e harmonia permitindo desempenhar um eficaz papel domestico no âmbito familiar, atribuindo características tipo emocionais, gentil, submissas e passivas (Deaux, 1985; Ferreira, 1995a, 1995b; Fernádez \& Vergara, 1998).

Desta forma, os papéis de gênero são expectativas relacionadas com os papéis que os indivíduos de cada sexo devem se comportar, assim, pode-se encontrar na sociedade um conjunto de idéias concernente à natureza da masculinidade e feminilidade; criaram-se, então, vários instrumentos intensificando a medição de tal construto, inicialmente elaboram-se um modelo unifatorial, assemelhando-se ao modelo biológico, considerando que os atributos psicológicos e comportamentais se diferenciam-se entre os sexos, tornando assim, excludentes tanto qualidades femininas quanto 
masculinas. Desta forma, masculinidade e feminilidade, são construtos hipotéticos representando um contínuo bipolar, no qual os homens ocupam o pólo masculino e as mulheres o feminino. (D'Amorim, 1989, 1997; Ferreira, 1995; Souza \& Ferreira, 1997).

A partir da década de setenta, com o movimento feminista, período de maior destaque para a mulher na sociedade; inúmeros pesquisadores intencionavam medir os conceitos de masculinidade e feminilidade, pois questionavam os valores dados as características femininas na mulher e as características masculinas no homem, rompendo assim, com a concepção paradigmática vigente na época, elabora-se assim, um modelo bifatorial para tal construto, o qual existem duas dimensões ortogonais e independentes, que ocorre no mesmo indivíduo (Ferreira, 1995; Cardoso \& Ferreira, 1995; Ortiz \& Valencia, 1998; Spence, Helmirh \& Stapp, 1975).

Com base neste modelo - o bifatorial - e com a perspectiva sócio-cultural de masculinidade e feminilidade distinguindo o papel instrumental e expressivo, surgem vários questionários que tentam medir tal construto, o questionário BSRI (Inventário de de Papéis Sexuais de Bem) destacado como o primeiro instrumento para medir esta nova concepção da identidade de gênero (Ferreira, 1995; Ortiz \& Valencia, 1998); segundo Huzt e Koller (1992) este questionário enfatiza algumas condições, tais como: as disposições dos indivíduos possuem empiricamente uma independência; entre as duas dimensões - masculinidade e feminilidade - existe um conceito de androginia que só poderá ser definido operacionalmente quando se obtém altos ou baixos níveis em ambas as dimensões; escores que apresentam alto tipificação sexual refletindo uma tendência a auto descrição concordando com os padrões comportamentais desejáveis por homens e mulheres.

Segundo Souza e Ferreira (1997) os recentes modelos teóricos sobre identidade de gênero incorporam pressupostos da teoria da aprendizagem social, a qual o indivíduo é influenciado pelos fatores ambientais na aquisição da identidade de gênero e do desenvolvimento cognitivo aportando por Kolberg, assim tanto os mecanismos cognitivos quanto os estágios de desenvolvimento são importantes como mediadores dessa identidade. Bem (1981) considera que tal identidade inicia-se a partir do momento em que a criança em desenvolvimento consegue distinguir os atributos, atitudes e comportamentos que estão vinculados ao sexo biológico, que através de associações apropriadas formará um esquema de gênero, que por sua vez, será responsável pelo processamento de novas informações sobre o gênero, adequando quando avaliado, ao esquema de acordo com sua preferência e atitudes, buscando uma aceitação social sobre tal escolha do papel sexual.

Desta forma, a identidade de gênero trata-se de um fenômeno aprendido, que sofre as mediações dos processos cognitivos e uma grande expectativa social desenvolvida em conformidade com os estereótipos sexuais, que são abstraídos a partir da experiência pessoal e social determinando os atributos e comportamentos típicos para cada sexo, bem como, ao papel desempenhado pelos agentes socializadores na sua formação (Souza \& Ferreira, 1997). À medida que se analise, principalmente este contexto social, muitos são os aspectos que se diferenciam, expondo questões em que homens e mulheres se adaptam aos indicadores ambientais supondo padrões sociais considerados inalterados (ver Hewstone, Strobe, Codol \& Stephenson, 1990).

Porém, para tal identidade destaca-se uma origem grupal, esta produzirá diferenças acessíveis e predizíeis no modo em que cada indivíduo se ver habitualmente a si mesmo e aos demais como parte do seu auto-esquema (Páez, Torres \& Echebarria, 1990; Smith \& Mackie, 1997); assim, a dimensão masculinidade e feminilidade diz respeito à 
identidade de gênero, referindo-se as expectativas da sociedade em relação à adequação da melhor regra a cada sexo em seu contexto sócio-cultural específico, enfatizando a conquista e harmonia pessoal que caracterizam o gênero em algumas culturas (Ferreira, 1995, 1997; Torres, Gomes, Techio \& Camino, 1996).

Desta forma, estas dimensões podem se configurar na saliência dos traços instrumentais e expressivos incorporados ao autoconceito do indivíduo, consistindo em construtos globais relacionados as variáveis psicológicas associadas ao gênero, tais como: estereótipos, valores e atitudes, capaz de se manifestar em todos os comportamentos intrínsecos aos papéis de gênero, centrado assim, na aprovação social (Ferreira 1997; Schwartz, 1994).

Este estudo tem como objetivos: 1 - compreender as dimensões de masculinidade e feminilidade em universitários, 2 - o grau de concordância entre homens e mulheres quanto a estas dimensões e 3 - através do MDS (Escala multidimensional) comprovar as dimensões deste construto.

\section{MÉTODO}

\section{Amostra}

A amostra foi composta de 423 estudantes universitários, do curso de ciências humanas das universidades publica e privada de João Pessoa - PB, de ambos os gêneros, $47 \%$ eram do sexo masculino e $53 \%$ do sexo feminino. A idade variava de 16 a 27 anos ( $M=$ 20,8; DP $=3,15)$. Esta amostra é não probabilística, podendo ser definida como intencional, pois se considerou a pessoa que, consultada, dispusera-se a colaborar respondendo o questionário que era apresentado.

\section{Instrumento}

Os sujeitos responderam a um instrumento que continha as seguintes questões:

Inventário de Papeis Sexuais de Bem, (BSRI, 1974). Este instrumento é composto por 18 adjetivos que avaliavam o grau de concordância ou discordância desempenhadas por cada gênero (masculino e feminino). Para respondê-lo a pessoa devia ler cada item e indicar o quanto está de acordo com o conteúdo expresso, utilizando para tanto uma escala de quatro pontos, tipo Likert, com os seguintes extremos: 1 = Nunca e $7=$ Sempre. Para assegurar tanto os itens como a compreensão das instruções que os antecediam, procedeuse a validação semântica, para tanto, foi considerada uma amostra de 20 sujeitos da população meta.

Caracterização Sócio-Demográfica. Uma folha separada foi anexada ao instrumento prévio, onde eram solicitadas informações de caráter sócio-demográfico (por exemplo, idade, sexo, estado civil, etc.).

\section{Procedimento}

O primeiro passo consistiu em definir um mesmo procedimento padrão que consistia em aplicar o instrumento coletivamente em sala de aula. Pessoas do grupo de pesquisa em comportamento político, treinadas e instruídas, ficaram responsáveis pela coleta dos dados. Após conseguir a autorização dos coordenadores dos cursos e do professor responsável pela disciplina, estes se apresentavam em sala de aula como solicitando a colaboração voluntária dos estudantes no sentido de responderem um questionário breve. Foi-lhes dito que não haviam respostas certas ou erradas, e que respondessem individualmente; a todos era assegurado o anonimato das suas respostas, que seriam tratadas em seu conjunto. Apesar do questionário ser auto-aplicável, contando 
com as instruções necessárias para que possam ser respondidos, os alunos cooperadores estiveram presentes durante toda a aplicação para retirar eventuais dúvidas ou realizar esclarecimentos que se fizessem indispensáveis. Um tempo médio de 15 minutos foram suficientes para concluir essa atividade.

\section{RESULTADOS E DISCUSSAO DOS DADOS}

Por meio de uma análise PC (Principais Componentes) e Rotação varimax e eigenvalue superior a 1,00, o uso desta técnica se mostrou meritória (KMO = ,71; Teste de Esfericidade de Bartlett, $\chi^{2}=678,92, p<0,001$ ) (ver Alzina,
1989; Bisquerra, 1989); foi analisada a distribuição dos fatores em relação ao Inventário de Papéis Sexuais, encontrando os 18 adjetivos, com apenas o adjetivo 17 (Submisso/a) atingindo um saturação baixa, apesar disto, foram obtido dois fatores bipolares: Fator 1 - Feminilidade, com Alpha de Crombach (a) de 0,69 e o fator 2 - Masculinidade apresentando o Alpha (a) de 0,68, tornando-os confiáveis estatiscamente e adequado para a análise dos principais componentes, como também, correspondendo as informações obtidas do Professor Dario Paéz.

Ambos os fatores dizem respeito à identidade de gênero em relação a melhor

Tabela1. Analise dos Principais Componentes do Inventário de Papéis Sexuais (BSRJ)

\begin{tabular}{|lcc|}
\hline Inventário de Papeis Sexuais - BSRI & Feminilidade & Masculinidade \\
\hline Caloroso/a, afetuoso/a & 0,62 & - \\
Carinhoso/a & 0,60 & - \\
Sensível as necessidades dos outros & 0,59 & - \\
Terno/a, delicado/a, suave & 0,55 & - \\
Compassivo/a (ter compaixão) & 0,55 & - \\
Compreensível & 0,48 & - \\
Amante das crianças & 0,51 & - \\
Egoísta & 0,36 & - \\
Chora facilmente & 0,26 & - \\
Atuam como líder & - & 0,64 \\
Dominante & - & 0,56 \\
Agressivo & - & 0,54 \\
Duro/a & - & 0,51 \\
Arriscar-se, gostar do perigo & - & 0,49 \\
Personalidade forte & - & 0,36 \\
Individualista & - & 0,30 \\
Atlético/a, desportivo/a & - & - \\
Submisso/a & - & 2,08 \\
\hline Engenvalue & 2,67 & 0,68 \\
\% variância Explicada & 0,69 & - \\
Alpha de Crombach ( $\alpha$ ) & & - \\
\hline
\end{tabular}

Notas: Carga fatorial $\geq 10$; Rotação varimax; eigenvalue $\geq 1,00$. 
regra que cada sexo, enfatiza na conquista e harmonia pessoal caracterizada no gênero, isto é, consiste em como se autopercebem seu papel vivencial (ver Paez, Vergara \& Achúcarro, 1995).

Após encontrar as dimensões de Masculinidade e Feminilidade, calculou-se um Qui-quadrado ( $\left.\mathrm{c}^{2}\right)$, com o intuito de encontrar diferença no que diz respeito à freqüência de homens e mulheres as dimensões de acima (Tabela 2). Desta forma, 54\% dos homens, significativamente, concordam com a Masculinidade, dispõem assim, as características de instrumentalidade desta dimensão; já 74\% das mulheres concordam com a Feminilidade, isto é, apresentam-se dispostas as características expressivas da dimensão.

Através de uma escala de análise multidimensional com todos os sujeitos $(N=423)$, atribuindo um escore $Z$, foi possível ter uma idéia a respeito das dimensões do BSRI encontradas (ver Figura 1), constatou-se que os itens do inventário se distribuem em duas dimensões, correspondendo a Masculinidade e Feminilidade, apresentando os seguintes indicadores de adequação da representação no espaço bidimensional: $R S Q=0,91$ e S-Stress $=0,14$.

Tabela 2. Freqüência de homens e mulheres universitários quanto aos indicadores de masculinidade e feminilidade.

\begin{tabular}{|lcccccc|}
\hline \multicolumn{7}{c|}{ Dimensão do BSRI } \\
\hline \multirow{2}{*}{ Gênero } & \multicolumn{2}{c|}{ Masculinidade } & \multicolumn{2}{c|}{ Femilidade } \\
\cline { 2 - 6 } & Discorda & Concorda & Discorda & Concorda & $\chi^{2}$ & $p<$ \\
\hline Mulher & 70 & 30 & 26 & 74 & 36,41 & 0,05 \\
Homem & 46 & 54 & 60 & 40 & 4,08 & 0,05 \\
\hline
\end{tabular}

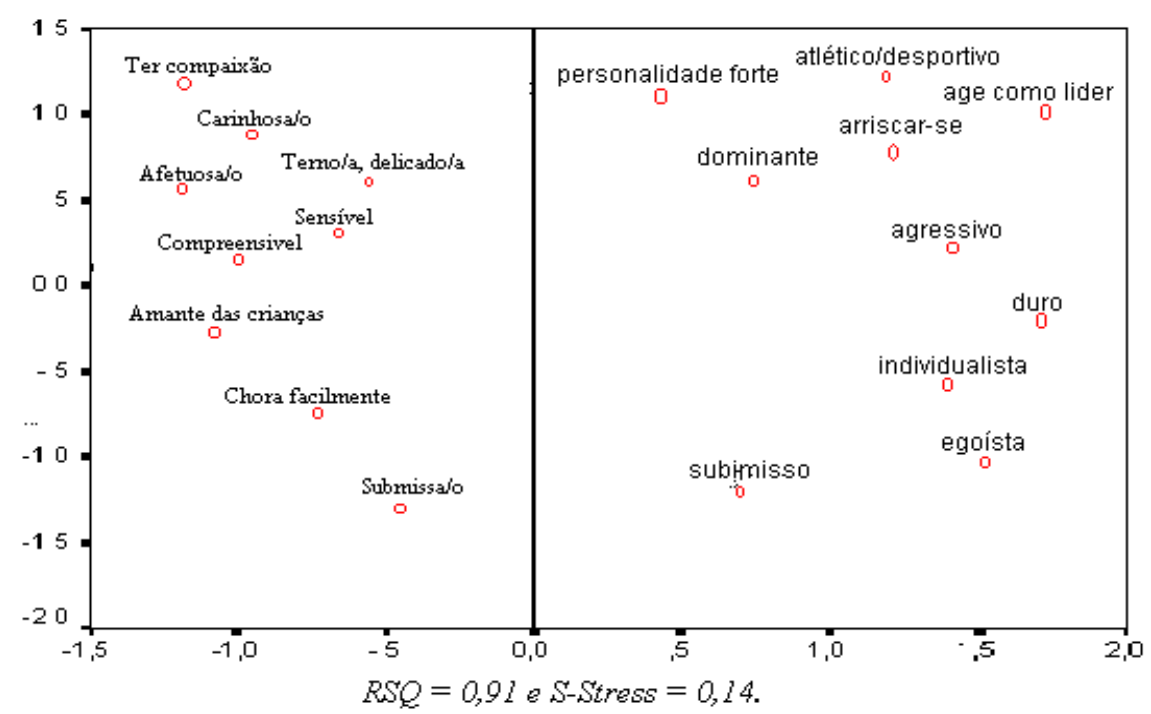

Figura 1. Escala Multidimensional do Inventário BSRI em Universitários de João Pessoa. 
Assim, é possível observar uma estrutura que situa os adjetivos que corresponde a Masculinidade no lado direito e o correspondente a Feminilidade no lado esquerdo da Figura 1, respectivamente. Vale destacar que, na análise dos principais componentes, os itens negativos encontrados o fator Feminilidade - egoísta $(-0,40)$ - e Masculinidade - Submisso/a ( - 0,13), apresentam-se como um componente inverso, correspondendo ao contrário do que diz o item na análise dos principais componentes, desta forma, através dessa análise - Análise Multidimensional - os itens se mostram correspondente ao seu fator de origem; egoísta corresponde a dimensão Masculinidade e submisso/a a de Feminilidade.

Assim, observa-se que na figura 1, a existência de duas dimensões devidamente caracterizadas, a primeira (lado direito da figura) Masculinidade, com os seguintes adjetivos: atlético e desportivo, personalidade forte, arriscar-se e gostar de perigo, atuar como líder, dominante, duro, agressivo, individualista, egoísta; e a segunda (lado esquerdo da figura) Feminilidade composta pelos adjetivos: Compassiva (ter compaixão), carinhosa, calorosa e afetuosa, terna, compreensível, sensível as necessidades dos outros, amante das crianças, chora facilmente, submissa.

\section{CONCLUSÃO}

A partir dos resultados obtidos concluise que o inventario mede estereótipos dos gêneros, indicado pelo contexto social e semântico das informações, tanto recebidas quanto respondidas pelo indivíduo, podendo estar relacionado as dimensões valorativas (ver Feather, 1984) nos quais são determinados pela inserção pessoal de cada sujeito na sociedade; assim, os sujeitos não somente pontuam as questões de acordo com sua experiência, como também, com o que lhe foi apresentado durante o seu desenvolvimento, fazendo com que se comporte baseado no melhor modelo correspondente na formação de gênero - masculino ou feminino - como proposto pela teoria acima. É importante chamar atenção para Tabela 2. Na freqüência do gênero às dimensões de masculinidade $\mathrm{e}$ feminilidade, as mulheres pontuam alto na concordância quanto à feminilidade, como também, na discordância na masculinidade, o mesmo se pode observar para os homens.

Pode-se, desta maneira, refletir que tal fenômeno se deve as amarras categoriais existentes na sociedade, ainda considerando a mulher como mais sensível ou frágil e os homens mais fortes, vigorosos etc.; levando em consideração tais questões, se observa que isto não condiz com as práticas observadas ultimamente na mídia, várias mulheres tem se destacado como atletas, líderes, etc., apresentando que não são papeis exclusivamente para homens; o mesmo pode se dizer para os homens, se mostrando como sensíveis, ternos e que se emocionam. Tais características, apresentam por meio de estudos desse tipo (ver Formiga, Gouveia, Maia \& Santos, 2000) que o ver e o agir das pessoas, isto é, opinar sobre tal fenômeno e fazer algo a seu respeito, principalmente quanto à discriminação feminina, têm sido inconstante. Poucos são os estudos direcionados as práticas intervencionistas sobre este fenômeno, principalmente, a de cunho científico. De acordo com Radice (1987; Borelli, 1998) as novas exigências ocorridas na sociedade, especialmente em relação aos valores culturais, estão levando a uma conscientização para as desigualdades existentes entre homem e mulher, que na maioria das vezes expressam concepções estereotipadas. Segundo Formiga, Santos, Gouveia e Leslie Júnior (2000; ver D'Amorim, 1997) tais considerações são de extrema importância, porém deve-se considerar tanto a estrutura quanto aos padrões valorativos capaz de promover práticas sociais menos discriminativas. 


\section{REFERÊNCIA BIBLIOGRAFICA}

ALZINA, R. B. (1989). Introducción conceptual al análises de dados multivariado: Un enfoque informático con los paquetes SPSS-X, BMDP, LISREL y SPAD. Barcelona: PPU.

BARON, R. (1996). Psicología. México: Prentice-Hall HispanoAmericana. $3^{\mathrm{a}}$ edição.

BEM, S. L. (1981). Gender schema theory: a cognitive account of sex typing. Psychological Review, 88, 354-364.

BISQUERRA, A. R. (1989). Introducción conceptual al análisis multivariable: Un enfoque informático con los paquetes SPSS-X, BMPD, LISREL Y SPAD. Barcelona: PPU.

BORELLI, A. (1998). Gênero: Desafios e perspectiva. Revista Unicsul, 4, 79-84.

CARDOSO, C. L. \& Ferreira, M. C. (1995). Identidade de gênero e satisfação conjugal. Psique revista, 5 (7).

D'AMORIM, M.A. (1989). Papel de gênero e atitudes acerca da sexualidade. Psicologia: Teoria e pesquisa, 5, 71-83.

D'AMORIM, M.A. (1997). Estereótipos de gênero e atitudes acerca da sexualidade em estudos sobre jovens brasileiros. Temas em Psicologia, 3, 121-134.

DEAUX, K. (1985). Sex and Gender. Annual Reveiw Psychological, 36, 49-81.

FERREIRA, M. C. (1995a). Masculinidade, feminilidade e ajustamento. Psicologia: Reflexão e Crítica, 8, 205-224.

FERREIRA, M. C. (1995b). Questionário Estendido de Atributos Pessoais: Uma medida de traços masculinos e femininos. Psicologia: Teoria de Pesquisa, 11 (2) 155-161.

FEATHER, N. T. (1984). Masculinity, femininity, psychological androgyny and the structure of values. Journal of Personality and Social Psychology, 47 (1), 604-620.
FERNÁNDEZ, I. \& Vergara, A. I. (1998). La dimensión de masculinidad-feminidad y los antecedentes, las reacciones mentales y los mecanismos de autocontrol emocional. RevisTA DE PSICOLOGÍA SOCIAL. 13 (2), 171-179.

FORMIGA, N. S., Gouveia, V. V., Andrade, J. M. Nascimento, A. S. \& Santos, M. N., (2000). Inventário de sexismo ambivalente: Sua adaptação ao contexto brasileiro. $V$ encontro mineiro de avaliação psicológica: Teorização e pratica e VIII conferência internacional de avaliação psicológica: Formas e contextos. Belo Horizonte: MG. 22 a 25 de agosto. (Resumos).

FORMIGA, N. S., Santos, M. N., Gouveia, V. V., Junior, L. L. C. \& Jesus, G. R. (2000). Prioridades valorativas e sexismo ambivalente: Considerações sobre as dimensões hostil e benévolo. XXX Reunião anual da sociedade brasileira de psicologia: SBP. Brasília: DF. 26 a 29 de outubro. (Resumos).

HEWSTONE, M. Stroebe, W., Codol, J. P. \& Stephenson,. G. M. (1990). Introducción a la psicología social: Una perspectiva europea. Barcelona: Editorial Ariel.

HUTZ, C. S. \& Koller, S. H. (1992). A mensuração do gênero: Uma readaptação do BSRI. Revista de Psicologia: Reflexão e crítica, 5 (2), 15-21.

MANSTEAD, A. S. R. \& Hewstone, M. (1995). The blackewell encyclopedia of social psychology. USA: Blackwell Publishers.

ORTIZ, G. \& Valencia, J. F. (1998). Identidad de genero en contexto: Un estudio empírico. Revista de psicología social, 13 (2), 251-259.

PÁEZ, D., Torres, B., \& Echebarria, A. (1990). Esquema de si, representación social y estereotipo sexual. Em G. Musitu (Org.), Procesos psicosociales básicos (pp. 229-234). Barcelona: PPU.

RADICE, J. (1987). Papéis sexuais no Nordeste do Brasil: Sua desejabilidade e 
possíveis conseqüências para a autorealização da mulher. Revista de Psicologia, 5, 93-103.

SOUZA, M. A., \& Ferreira, M.A.C. (1997). Identidade de gênero masculina em civis e militares. Psicologia: Reflexão e Crítica, 10, 301-314.

SCHWARTZ, S. H. (1994). Beyond individualism/collectivism: New cultural dimensions of values. Em: U. Kin, H. C. Triandis, Ç. Kagitçibasi, S-C. Choi \& G Yoo (eds.). Individualism and collectivism: theory, method and applications. (pp. 85-122) Thousand Oaks, CA: Sage Publications.
SPENCE, J. T., Helmreich, R., \& Stapp, J. (1975). Ratings of self and peers on sex role attributes and their relation to self-esteem and conceptions of masculinity and feminility. Journal of Personality and Social Psychology, 32, 29-39.

TORRES, A. R., Gomes, G. O. Techio, E. M. \& Camino, L. (1996). Diferenças entre sociedades individualistas e coletivistas relacionadas com o fenômeno da diferenciação intergrupal. Em: L. Lhullier, L. Camino \& S. Sandoval (org.). Estudos sobre comportamento político: teoria e pesquisa. Florianópolis: Letras contemporâneas. 